\title{
MLH1 Exon 12 Gene Deletion Leading to Lynch Syndrome: A Case Report
}

\author{
Shiyun Cui ${ }^{a}$ Xiao Zhang ${ }^{b}$ Ruihan Zou ${ }^{b}$ Fan Ye ${ }^{b}$ Yutong Wang ${ }^{b}$ Jing Sun ${ }^{a}$ \\ ${ }^{a}$ Department of Oncology, The First Affiliated Hospital of Nanjing Medical University, Nanjing, China; \\ ${ }^{b}$ The First School of Clinical Medicine, Nanjing Medical University, Nanjing, China
}

\section{Established Facts}

- The most common causes of Lynch syndrome (LS) include nonsense and frameshift mutations. These mutations lead to termination codons in mismatch repair (MMR) genes, followed by a large number of deletions or insertions.

- Data from the NCCN guidelines support the use of checkpoint inhibitor therapy as first-line treatment for deficient MMR (dMMR) CRC. The NCCN guidelines also state that dMMR is a therapeutic biomarker for checkpoint inhibitor therapy and this therapy should be applied to dMMR patients only.

\section{Novel Insights}

- In this report, we demonstrate that c.1057_1060delGCTG, an MLH1 gene mutation never reported before, can result in abnormal splicing of the MLH1 gene during the development of LS. This patient died of multiple organ failure soon after undergoing checkpoint inhibitor therapy. We recommend checkpoint inhibitor therapy as the first-line treatment for LS patients.

\section{Keywords}

Lynch syndrome - Colorectal cancer - Mismatch repair .

Germline mutation PD-1 inhibitors

\section{Abstract}

Introduction: Deleterious heterozygous mutation of the MLH1 gene is an important cause of Lynch syndrome (LS), an autosomal dominant cancer caused by functional defects in the DNA mismatch repair (MMR) complex. Case Report: The proband was a 35-year-old patient with confirmed colorectal cancer (CRC). Immunohistochemical (IHC) staining revealed the absence of MLH1 and PMS2 expression in the colorectal tissue specimens of the patient. Genetic counselling and tumor gene testing were performed using nextgeneration sequencing technology. The genetic tumor verification report showed the deletion of 4 bases in exon 12 of the tested MLH1 gene and a transcoding mutation. To our knowledge, this germline splice site mutation of MLH1 has not been reported before. The proband accepted several therapeutic regimens including PD-1 inhibitor and ultimately died of multiple organ failure. Conclusion: Nonsense mutations and frameshift mutations of MMR genes are the most common causes of LS. Common mutations include those in MSH2, MLH1, MSH6, and PMS2. We report a mutation of $\mathrm{MLH} 1$ that has never been reported before. We recommend that patients with a history of colon or rectal cancer receive universal MMR or MSI testing and checkpoint inhibitor therapy for the first-line treatment of deficient MMR CRC.

(C) 2021 The Author(s)

Published by S. Karger AG, Basel

Shiyun Cui and Xiao Zhang should be regarded as joint first authors for their equal contributions. 


\section{Introduction}

Lynch syndrome (LS; hereditary nonpolyposis colorectal cancer [CRC]) is one of the most common causes of CRC. Hereditary CRC syndrome has a high penetrance and low incidence, accounting for $5 \%$ of CRC cases diagnosed annually [1]. However, many carriers with a high risk of disease have not been identified, and it is estimated that in the USA, with appropriate screening, approximately 12,000 people can be diagnosed with hereditary nonpolyposis colon cancer (HNPCC) each year [2].

LS is acquired through autosomal dominant inheritance and it is caused by a germline mutation of one of the mismatch repair (MMR) genes. Common mutations
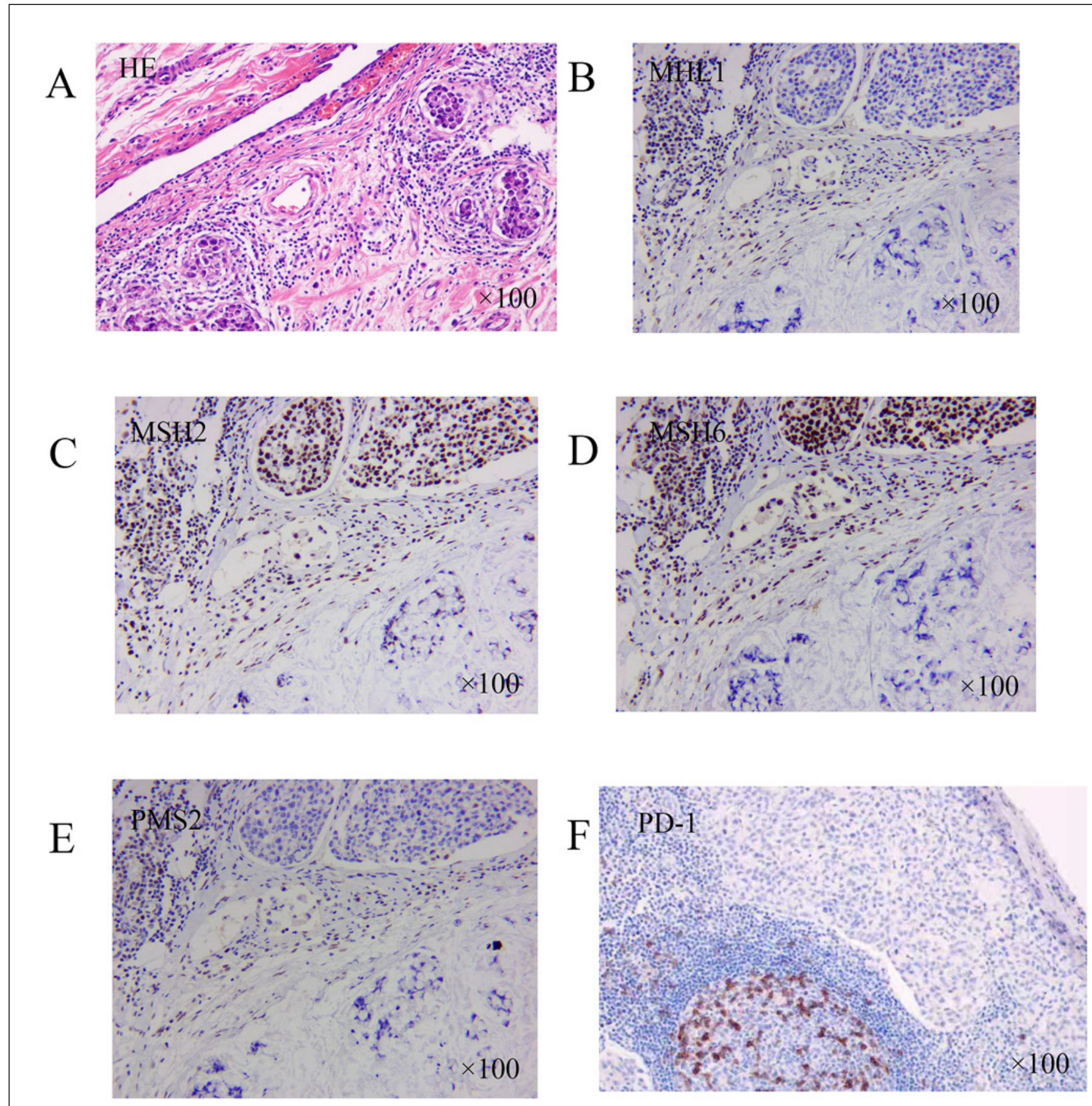

Fig. 1. Hematoxylin-eosin (HE) staining and IHC staining of colon cancer tissue specimens. Magnification $\times 100$. A HE staining. B Negative MHL1 expression. C Positive MSH2 expression. D Positive MSH6 expression. E Negative PMS2 expression. F IHC staining of PD-1. 
include those in MSH2 [3], MLH1 [4], MSH6 [5], and PMS2 [6]. The MLH1-encoded protein can be heterodimerized to form MutL- $\alpha$ with the MMR endonuclease PMS2, which is part of the DNA MMR system [7]. When MutL binds to MutS and some helper proteins, the PMS2 subunit of MutL introduces a single-strand fracture near the DNA mismatch, providing an entry point for exonuclease degradation. The encoded proteins also participate

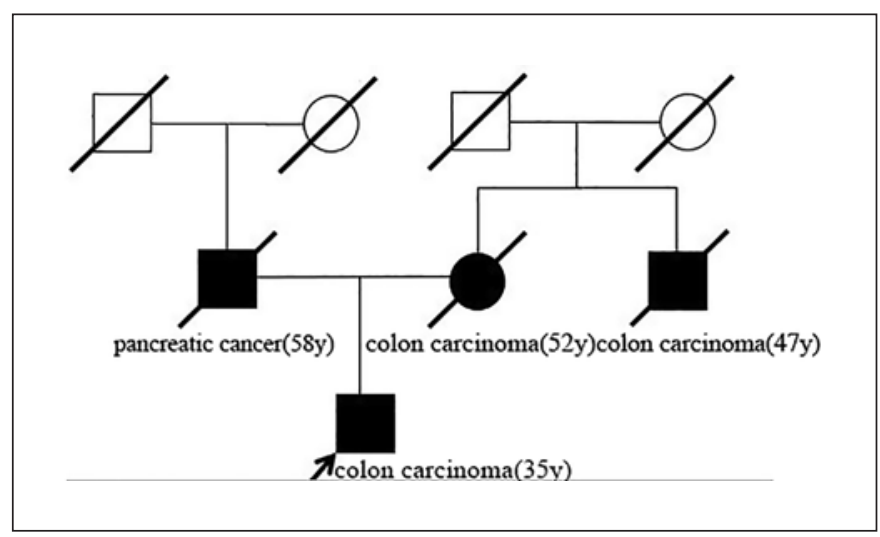

Fig. 2. Pedigree of the patient. The arrow indicates the proband. Squares and circles denote males and females, respectively. Closed symbols indicate persons with cancer. in DNA damage signal transduction and can form MutL, which is involved in meiosis after heterodimerizing with the DNA MMR protein MLH3. These MMR genes are thought to be a common site of mutations in HNPCC. Here, we used second-generation high-throughput sequencing technology to analyze variants (including point mutations and deletion/insertion mutations) in exons of genes related to inherited digestive tract tumors and their adjacent 10-bp intron regions (within $20 \mathrm{bp}$ ) in the proband and found a mutation of MLH1 that had not been reported previously. The patient refused to disclose private information, so we anonymized the information. This case report was prepared following CARE guidelines [8].

\section{Case Report}

The patient underwent radical resection of colon cancer at the age of 35 years on January 12, 2018, at our hospital. Pathological detection revealed poorly differentiated cancer, and the cancer tissue infiltrated the entire wall of the intestine to the serous layer (Fig. 1a). The pathological stage was pT4a pN2b pMx IIIC. The family tree is shown in Figure 2. His father was found to have pancreatic cancer at the age of 58 years, but he had no history of colorectal or stomach cancer. His mother and maternal uncle developed colon carcinoma at the age of 52 and 47 years, respective-

Fig. 3. CT images of the patient. A First progression. B Second progression.

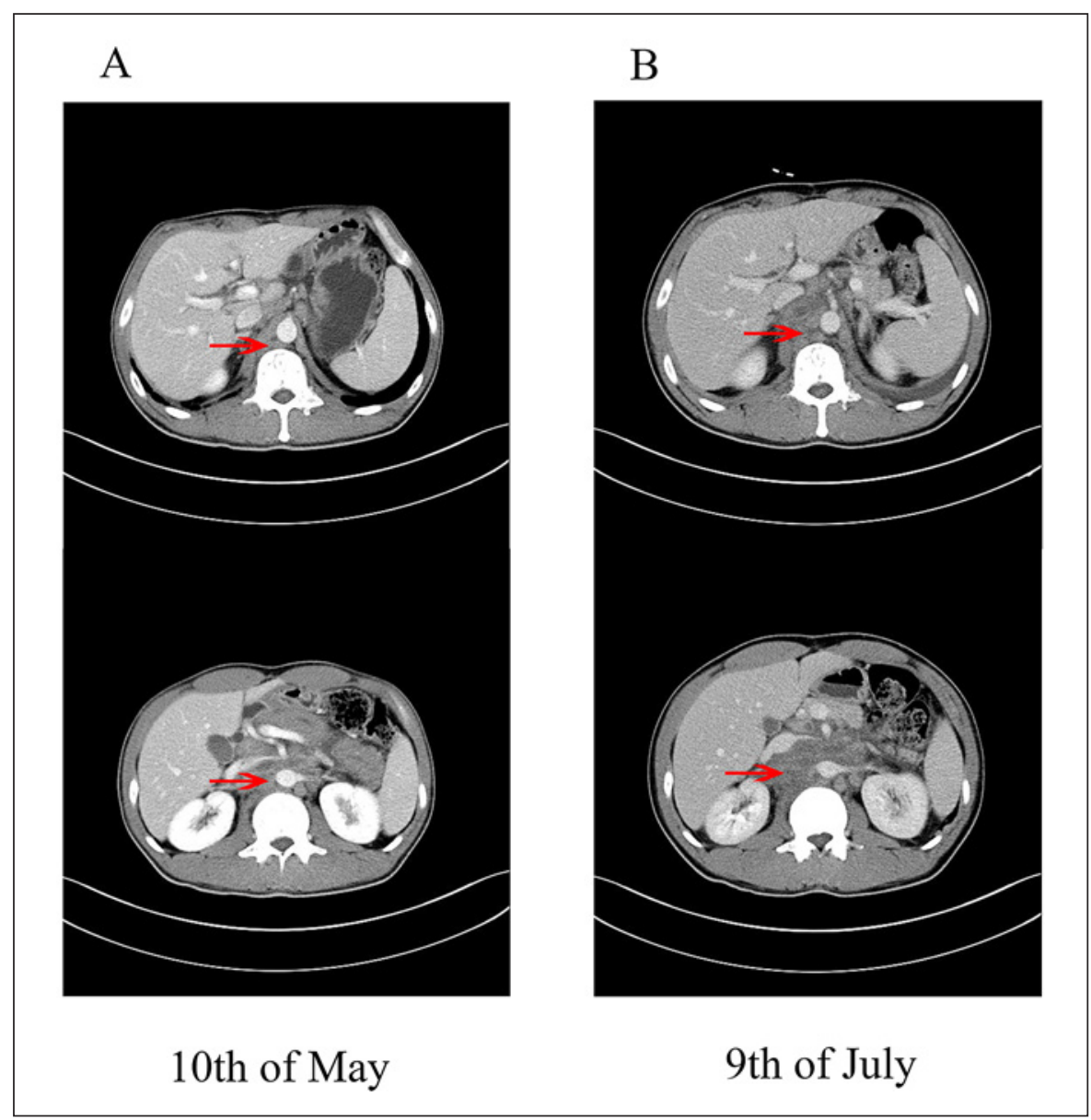




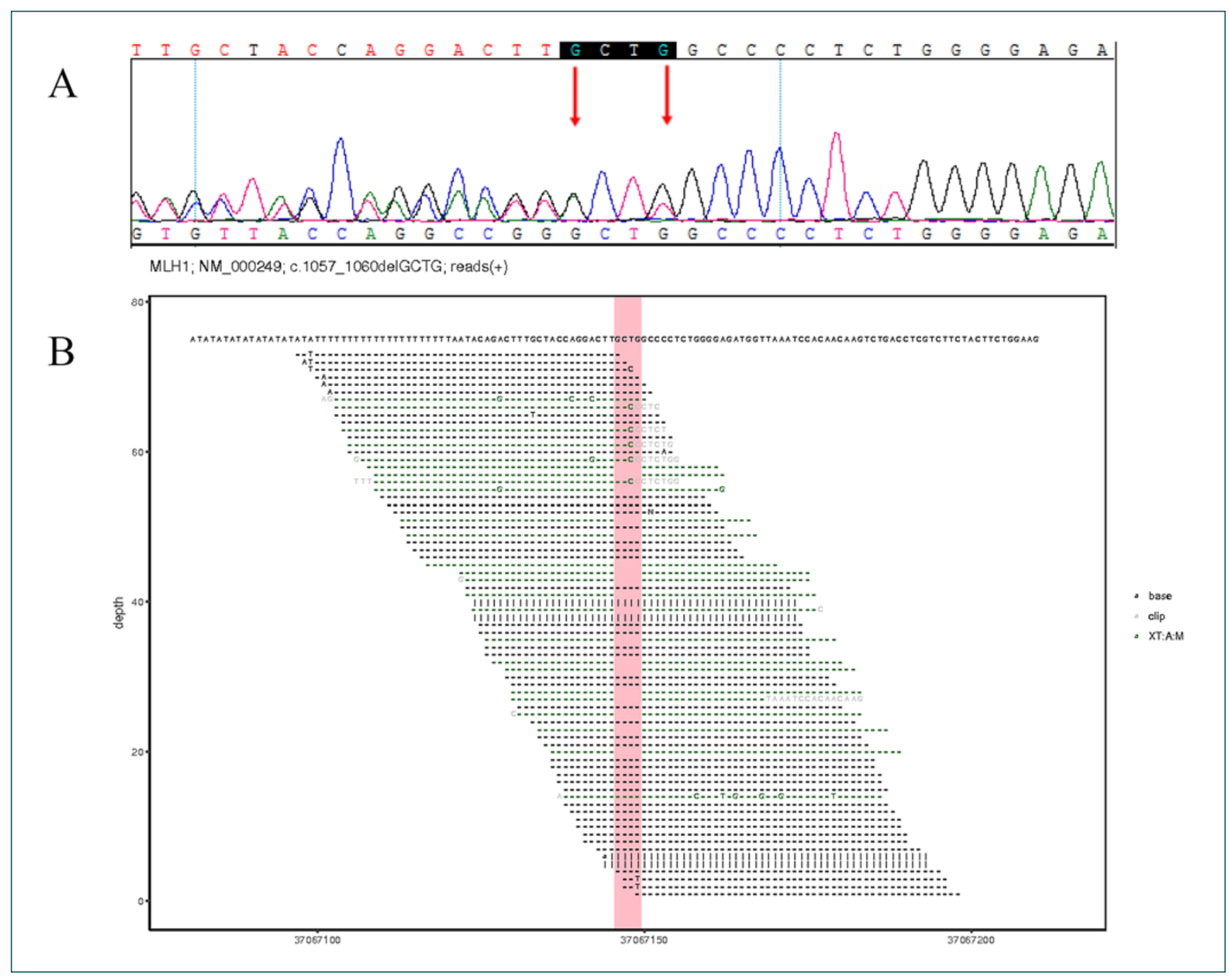

Fig. 4. A Results of gene detection (Sanger sequencing). The frameshift mutation c.1057_1060delGCTG (p.gly354 profs *12) was detected in the MHL1 gene of the proband (arrows). B Outcomes of high-throughput genome sequencing; the red vertical line represents gene mutation sites.

ly. The patient accepted oxaliplatin $(225 \mathrm{mg}$ on day 1$)$ and capecitabine ( 3 tablets b.i.d. on days 1-14) for 4 cycles as postoperative therapy.

After 4 cycles of chemotherapy, the patient's condition was good, but examination of tumor markers from the serum on April 16,2018 , implied cancer progression. The CEA level was $33.38 \mathrm{ng} /$ $\mathrm{mL}$ (it should be $<4.70 \mathrm{ng} / \mathrm{mL}$ ). A CT scan performed on May 10 , 2018, also revealed the progression of cancer (Fig. 3a). Examination of CEA and CA724 levels verified progression, with a CEA level of $390.50 \mathrm{ng} / \mathrm{mL}$ and a CA724 level exceeding $300 \mathrm{U} / \mathrm{mL}$. Because he met the criteria of the revised Bethesda guidelines for LS screening, the patient was referred for genetic counselling and provided written informed consent for the genetic analysis. The expression of MLH1, MSH2, MSH6, and PMS2 proteins in the resected tumor was analyzed by immunohistochemistry. Immunohistochemical (IHC) staining demonstrated the loss of MLH1 and PMS2 expression in colon cancer tissue specimens obtained from the patient (Fig. 1b, e) and revealed deficient MMR (dMMR). Then, we used second-generation high-throughput sequencing technology to analyze variants (including point mutations and de- letion/insertion mutations) in the exons of genes related to hereditary digestive tract tumors and their adjacent 10-bp intron region variants (within $20 \mathrm{bp}$ ) in the proband and found the frameshift mutation c.1057_1060delGCTG (p.gly354profs *12) (Fig. 4b). Sanger sequencing was performed to verify the mutation (Fig. 4a). Four bases in exon 12 of the tested MLH1 gene were deleted, and a frameshift mutation occurred at position 365 , which led to early termination of the coding protein and resulted in a truncated polypeptide chain; the normal gene encodes 756 amino acids. The functional and clinical significance of this mutation has not been reported in the literature. The same mutation was also found in blood samples of the patient's mother and uncle but not in the patient's aunt, who appeared to be cancer free (Fig. 5).

According to NCCN guidelines, we recommended PD-1 inhibitors for treatment [9]. IHC staining of PD-1 is shown in Figure 1f. The patient refused treatment because of his financial status and alternatively accepted second-line chemotherapy (bevacizumab, $300 \mathrm{mg}$ i.v.d. on day 1, irinotecan hydrochloride at $300 \mathrm{mg}$ i.v.d. on day 2 , and $5-\mathrm{FU}$ at $0.5 \mathrm{~g}$ on day $1+3.5 \mathrm{~g}$ on day $2 ; 46 \mathrm{~h}$ i.v.d.) [10] beginning on May 17, 2018. The CT scan on July 9, 2018, re- 
MLH1 all.seq $(1>79540)$

$\nabla$ 18B3193596-0285-D05_MLH1Cds12R $(14>357) \leftarrow$

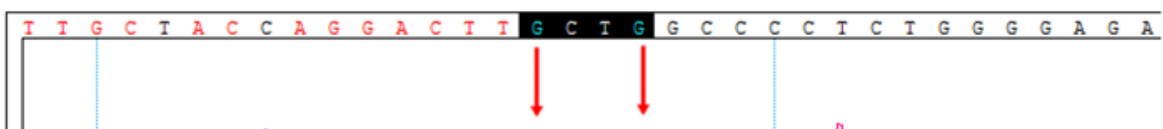

A

$\nabla$ 18B3208435-0404-E03_MLH1cd312R(16>339)

B

$\nabla$ 18B3208436-0404-F03_MLH1Cds12R (14>479)

$\mathrm{C}$

$\nabla$ 18B3212928-1-0404-G03_MLH1cds1 (18>445)

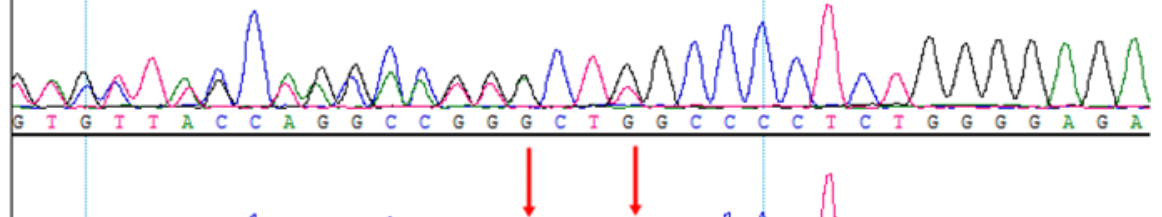

西

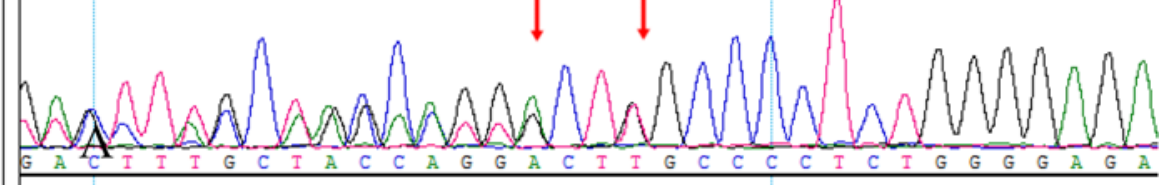

$\mathrm{D}$

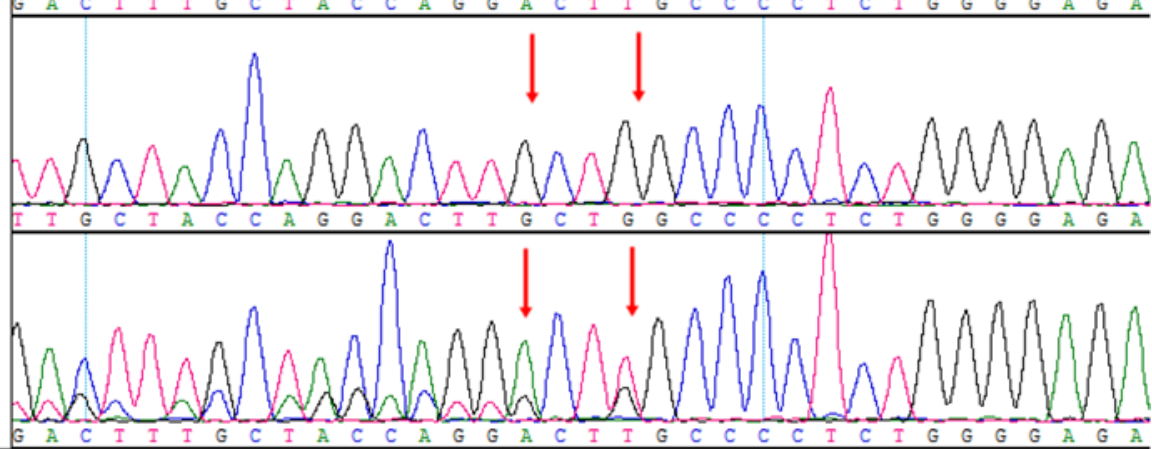

MLH1; NM_000249; c.1057_1060delGCTG; p.Gly354Profs*12

Fig. 5. The results of family gene detection were analyzed by Sanger sequencing. A Heterozygous (Het) mutations were observed in the proband. B Het mutations were observed in the proband's uncle. C Genetic tests on the proband's maternal aunt showed normal results. D Het mutations were observed in the proband's mother. The red arrows show the Het mutation c.1057_1060delGCTG (p.gly354profs *12).

Table 1. Organization of the case into a timeline

\begin{tabular}{|c|c|}
\hline Date & {$[\mathrm{T}] /[\mathrm{S}] /[\mathrm{E}]$} \\
\hline January 12, 2018 & [T] Radical resection of colon cancer \\
\hline January 2018 & [E] Pathological detection \\
\hline February 2018 & [T] Four cycles of chemotherapy (oxaliplatin and capecitabine) \\
\hline April 16, 2018 & $\begin{array}{l}\text { [E] Six items of tumor markers from serum (AFP, CEA, CA199, CA724, CYFRA21-1, } \\
\text { and NSE), CEA } 33.38 \mathrm{ng} / \mathrm{mL} \text {, NSE } 22.67 \mathrm{ng} / \mathrm{mL}\end{array}$ \\
\hline May 10, 2018 & $\begin{array}{l}\text { [E] CT detection revealed progression } \\
{[E] \text { Tumor markers from serum, CEA } 390.50 \mathrm{ng} / \mathrm{mL}, \text { CA724 exceeded } 300 \mathrm{U} / \mathrm{mL}}\end{array}$ \\
\hline May 2018 & $\begin{array}{l}\text { [E] Immunohistochemical staining, loss of MLH1 and PMS2 expression } \\
\text { [E] Second-generation high-throughput sequencing and the Sanger method revealed the } \\
\text { frame-shift mutation c.1057_1060delGCTG (p.gly354profs }{ }^{\star} 12 \text { ) }\end{array}$ \\
\hline May 17, 2018 & [T] Chemotherapy (bevacizumab, irinotecan hydrochloride, and 5-FU) \\
\hline July 9, 2018 & $\begin{array}{l}\text { [E] CT scan revealed progression } \\
\text { [E] Tumor markers from serum, CEA } 411.80 \mathrm{ng} / \mathrm{mL}, \text { CA724 } 253.50 \mathrm{U} / \mathrm{mL}\end{array}$ \\
\hline July 16, 2018 & [T] PD-1 inhibitors therapy \\
\hline July 23, 2018 & [S] The patient died of multiple organ failure \\
\hline
\end{tabular}

[T], treatment; [S], symptoms; [E], examination. 
vealed progression again (Fig. 3b), consistent with the elevated levels of CEA and CA724, which were $411.80 \mathrm{ng} / \mathrm{mL}$ and $253.50 \mathrm{U} /$ $\mathrm{mL}$, respectively. The patient finally accepted PD-1 inhibitor therapy (pembrolizumab at $200 \mathrm{mg} \mathrm{q} 3 \mathrm{w}$ ) on July 16, 2018. However, 1 week later, the therapy failed to stop tumor progression and the patient died of multiple organ failure (Table 1).

\section{Discussion}

The most common causes of LS include nonsense mutations and frameshift mutations, which lead to the early appearance of termination codons in MMR genes, followed by a large number of deletions or insertions [11]. The spliceosome $\left(5^{\prime}\right.$-gctg- $\left.3^{\prime}\right)$ affects the splicing of mRNA, causing a change in the peptide chain from Gly at 354 and termination of the peptide chain at 365 (it should have been 756 amino acids). In this report, we demonstrated that c.1057_1060delGCTG resulted in abnormal splicing of the MLH1 gene. Since this genetic change is not a highly conserved base mutation at the regular position of gt-ag (the intron starts with the dinucleotide "gt" and ends with the dinucleotide "ag" - the so-called gt-ag rule), we evaluated the effects of this 4-bp deletion on splicing. According to the prediction, skipping exon 12 will lead to a frameshift and eventually stop translation at exon 12 , which is consistent with our detection results. The members of this family were subjected to predictive tests. Regardless of the family history of gastric cancer, all carriers accepted the HNPCC monitoring plan each year (starting from the age of 25 years, but the earliest tumor in the family was diagnosed at least 5 years before onset), which included the following: annual check-up, colonoscopy, abdominal ultrasound, colposcopy (in the Department of Gynecology), ultrasound, and gastroscopy. The MLH1-encoded protein can heterodimerize with the MMR endonuclease PMS2, which is part of the DNA MMR system, to form MutL- $\alpha$. The combined loss of MLH1 and PMS2 is characteristic of patients with MLH1 mutations, while isolation and deletion of the PMS2 protein in tumor tissue indicates a germline mutation of PMS2 [7].

According to NCCN guidelines for colon cancer (https://www.nccn.org/), it is recommended that all patients with a personal history of colon or rectal cancer undergo universal MMR or MSI (microsatellite instability) testing. In addition to its role as a predictive marker for immunotherapy use in the advanced CRC setting, MMR/MSI status can also help to identify individuals with LS.

Limited data referred to by the NCCN guidelines support the use of checkpoint inhibitor therapy for the firstline treatment of dMMR CRC. An abstract on the phase II CheckMate-142 trial reported results for checkpoint inhibitors in 45 patients with previously untreated dMMR CRC [12]. The objective response rate (ORR) was found to be $60 \%$ (95\% CI $44.3-74.3 \%$ ), with a median follow-up of 13.8 months. After 19.9 months of follow-up, the investigator-assessed ORR was 64\% (95\% CI 49-78\%), the disease control rate was $84 \%$ (95\% CI 71-94\%), and the duration of response was not reached. Pembrolizumab has been approved by the FDA to treat MSI-H or dMMR solid tumors because of the $39.6 \%$ ORR among 149 patients with 15 different tumor types (95\% CI 31.7-47.9), with a $7 \%$ complete response rate [13]. A report revealed a case in which 1 MSI-H CRC patient progressed after checkpoint inhibitor therapy and accepted combination immunotherapy with nivolumab plus ipilimumab. This therapy has been proven to be successful in metastatic melanoma patients and controls progression. This case may have reference significance for future treatment [14]. The NCCN guidelines also state that dMMR status is a biomarker for checkpoint inhibitor therapy and emphasize that this therapy should be applied to dMMR patients only. A review summarized relevant studies to confirm this view [15]. An effective therapeutic regimen is urgently required for non-dMMR/MSI-H patients (95\% in the metastatic setting) [16]. LS is caused by a germline mutation of the MMR gene, which could result in dMMR. Therefore, checkpoint inhibitor therapy is a good choice for LS patients, as revealed by genetic analyses. In this case, the best time for the patient to accept PD-1 inhibitor therapy was after the genetic analysis, which revealed dMMR. If the patient had accepted PD-1 inhibitor therapy sooner, lethal progression might not have occurred.

Although the patient died of multiple organ failure, we cannot exclude the possibility of disease overprogression (HPD). This concept was first defined in a study by French scholars, who reported that the tumor growth rate after immunotherapy was more than twice as high as that before treatment, with an incidence of 9\% [17]. Patients with certain mutations, such as MDM2 gene amplification [18], are at risk of HPD. We hypothesize that the death of the patient in this case might have been related to HPD, but we do not have gene detection results; therefore, the results cannot be verified.

To our knowledge, the mutations detected in this study have not been previously reported. In addition, at the date of writing, these mutations had not been included in the International Society for Gastrointestinal Inherited Tumor database (https://www.insight-group.org/) or in the Human Gene Mutation Database (http://www. hgmd.org/).

\section{Statement of Ethics}

This study protocol was reviewed and approved by the Ethics Committee of The First Affiliated Hospital of Nanjing Medical University (approval No. 2021-QT-07). Written informed consent was obtained from the participants prior to this study. 


\section{Conflicts of Interest Statement}

The authors have no conflict of interests to declare.

\section{Funding Sources}

This study was supported by a grant from the National Natural Science Foundation of China (NSFC 82073164, 82072721).

\section{Author Contributions}

Jing Sun: acquisition of funding and general supervision of a research group. Shiyun Cui: acquisition of funding and general administrative support. Xiao Zhang: editing. Ruihan Zou: writing assistance. Fan Ye: technical editing. Yutong Wang: language editing.

\section{References}

1 Smith RA, Manassaram-Baptiste D, Brooks D, Cokkinides V, Doroshenk M, Saslow D, et al. Cancer screening in the United States, 2014: a review of current American Cancer Society guidelines and current issues in cancer screening. CA Cancer J Clin. 2014 JanFeb;64(1):30-51.

2 Kwak EL, Chung DC. Hereditary colorectal cancer syndromes: an overview. Clin Colorectal Cancer. 2007 Jan;6(5):340-4.

3 Truninger K, Menigatti M, Luz J, Russell A, Haider R, Gebbers JO, et al. Immunohistochemical analysis reveals high frequency of PMS2 defects in colorectal cancer. Gastroenterology. 2005 May;128(5):1160-71.

4 Arnold S, Buchanan DD, Barker M, Jaskowski L, Walsh MD, Birney G, et al. Classifying MLH1 and MSH2 variants using bioinformatic prediction, splicing assays, segregation, and tumor characteristics. Hum Mutat. 2009 May;30(5):757-70.

5 Lawes DA, Pearson T, Sengupta S, Boulos PB. The role of MLH1, MSH2 and MSH6 in the development of multiple colorectal cancers. Br J Cancer. 2005 Aug;93(4):472-7.

6 de Jong AE, van Puijenbroek M, Hendriks Y, Tops C, Wijnen J, Ausems MG, et al. Microsatellite instability, immunohistochemistry, and additional PMS2 staining in suspected hereditary nonpolyposis colorectal cancer. Clin Cancer Res. 2004 Feb;10(3):972-80.
7 Rahner N, Friedrichs N, Wehner M, Steinke V, Aretz S, Friedl W, et al. Nine novel pathogenic germline mutations in MLH1, MSH2, MSH6 and PMS2 in families with Lynch syndrome. Acta Oncol. 2007;46(6):763-9.

8 Riley DS, Barber MS, Kienle GS, Aronson JK, von Schoen-Angerer $\mathrm{T}$, Tugwell $\mathrm{P}$, et al. CARE guidelines for case reports: explanation and elaboration document. J Clin Epidemiol. 2017 Sep;89:218-35.

9 Le DT, Uram JN, Wang H, Bartlett BR, Kemberling $\mathrm{H}$, Eyring $\mathrm{AD}$, et al. $\mathrm{PD}-1$ blockade in tumors with mismatch-repair deficiency. N Engl J Med. 2015 Jun;372(26):2509-20.

10 Hurwitz HI, Tan BR, Reeves JA, Xiong H, Somer B, Lenz HJ, et al. Phase II randomized trial of sequential or concurrent FOLFOXIRI-bevacizumab versus FOLFOX-bevacizumab for metastatic colorectal cancer (STEAM). Oncologist. 2019 Jul;24(7):92132.

11 Lynch HT, Lynch PM, Lanspa SJ, Snyder CL, Lynch JF, Boland CR. Review of the Lynch syndrome: history, molecular genetics, screening, differential diagnosis, and medicolegal ramifications. Clin Genet. 2009 Jul;76(1):1-18.

12 Lenz HJ, Lonardi S, Zagonel V, Cutsem EV, Limon ML, Wong KY, et al. Nivolumab (NIVO) + low-dose ipilimumab (IPI) as firstline $(1 \mathrm{~L})$ therapy in microsatellite instability-
high/DNA mismatch repair deficient (MSIH/dMMR) metastatic colorectal cancer (mCRC): clinical update. J Clin Oncol. 2019;37(15):3521-21.

13 Marcus L, Lemery SJ, Keegan P, Pazdur R. FDA approval summary: pembrolizumab for the treatment of microsatellite instabilityhigh solid tumors. Clin Cancer Res. 2019 Jul;25(13):3753-8.

14 Das S, Allen A, Berlin J. Immunotherapy After Immunotherapy: Response rescue in a patient with microsatellite instability-high colorectal cancer post-pembrolizumab. Clin Colorectal Cancer. 2020 Jun;19(2):137-40.

15 Jácome AA, Eng C. Role of immune checkpoint inhibitors in the treatment of colorectal cancer: focus on nivolumab. Expert Opin Biol Ther. 2019 Dec;19(12):1247-63.

16 Stein A, Folprecht G. Immunotherapy of colon cancer. Oncol Res Treat. 2018;41(5):2825.

17 Champiat S, Dercle L, Ammari S, Massard C, Hollebecque A, Postel-Vinay S, et al. Hyperprogressive disease is a new pattern of progression in cancer patients treated by antiPD-1/PD-L1. Clin Cancer Res. 2017 Apr; 23(8):1920-8.

18 Burgess A, Chia KM, Haupt S, Thomas D, Haupt Y, Lim E. Clinical overview of MDM2/X-targeted therapies. Front Oncol. 2016 Jan;6:7. 